\section{OPTICAL PARAMETRIC OSCILLATORS}

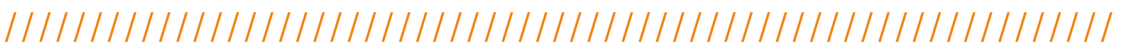

Jean-Michel MELKONIAN, Jean-Baptiste DHERBECOURT,

Myriam RAYBAUT, Antoine GODARD*

DPHY, ONERA, Université Paris-Saclay, 91123 Palaiseau, France

*antoine.godard@onera.fr
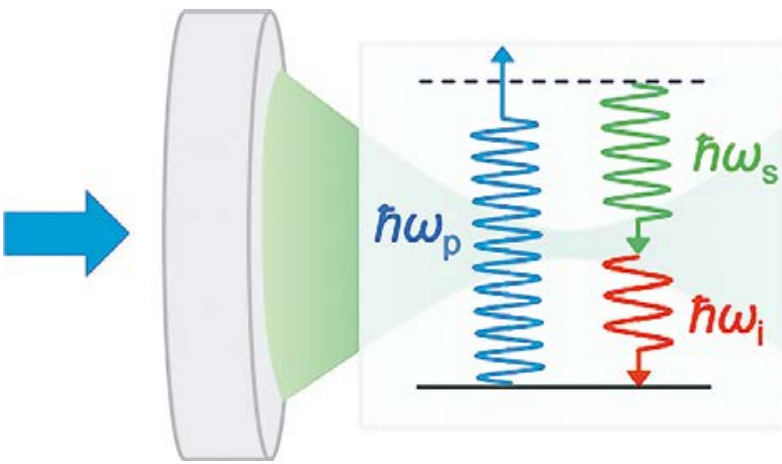

Optical parametric oscillators are versatile devices for generating a tunable coherent radiation from an incident laser beam. They rely on nonlinear frequency conversion to cover spectral ranges that are poorly or not covered by direct laser emission. This article presents a short overview of the optical parametric oscillators principles of operation, main implementations and related applications.

https://doi.org/10.1051/photon/202111053

This is an Open Access article distributed under the terms of the Creative Commons Attribution License (https://creativecommons.org/licenses/by/4.0), which permits unrestricted use, distribution, and reproduction in any medium, provided the original work is properly cited.

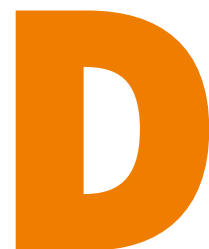
espite the various developments of wavelength tunable lasers, their spectral coverage remains incomplete, especially when a high peak power is required. Nonlinear optical processes, which arise from the non-harmonical response of electric dipoles in dielectric media when submitted to an intense electrical field, allows to convert the frequency emitted by a laser to another frequency, while preserving various properties of the original laser emission, namely its temporal and spatial coherences. More interestingly, the generated frequencies can be tuned by controlling the so-called phasematching condition, which relies on the dependence of the indices of refraction upon the crystallographic orientation, temperature, light polarization, and even mechanical strain.

Optical parametric generators (OPGs) and oscillators (OPOs) are devices

\section{Optical Parametric Oscillator}

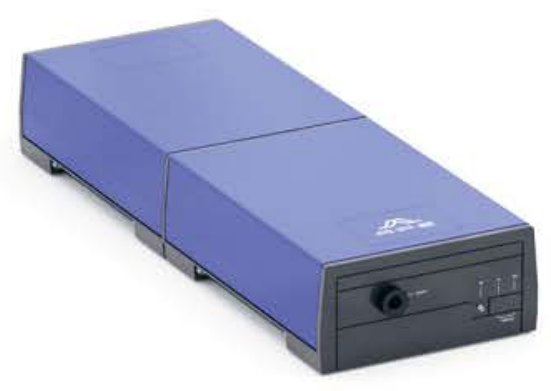

Automated wavelength tuning

- For femto- \& picosecond laser

- Suitable for Ti:Sa or Yb laser

Output wavelength 505 ... 4800 nm

- Extendable (SHG, THG, FHG \& DFG)

- Integrated spectrometer \& diagnostics
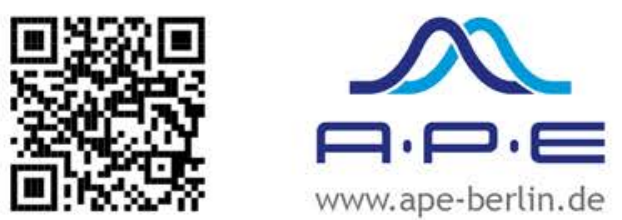


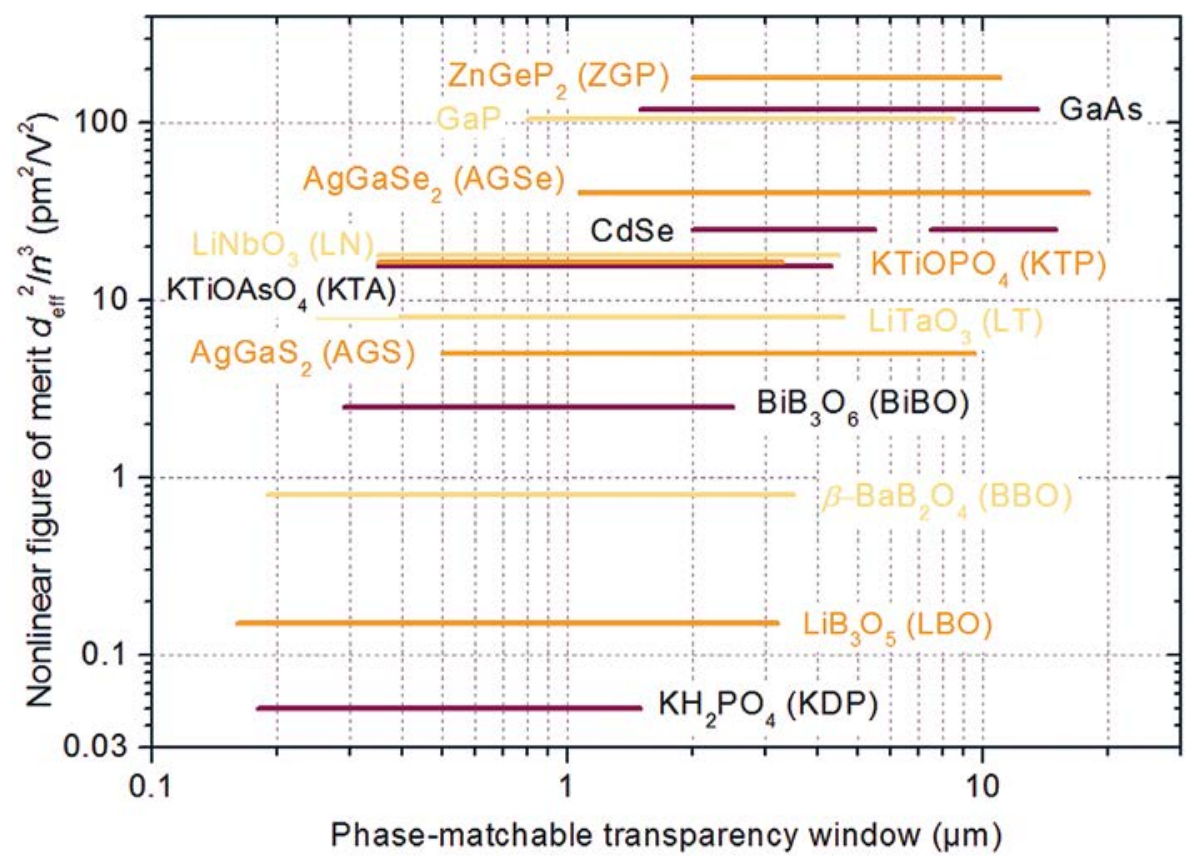

that efficiently generate such tunable coherent radiation from an incident laser beam.

\section{OPTICAL PARAMETRIC}

\section{AMPLIFICATION AND GENERATION}

Parametric interaction is a threewave mixing phenomenon arising in a non centro-symmetric medium, such as a crystal (figure 1). The most intense beam, with the highest angular frequency $\omega_{p}$, is called the pump. When the signal wave of frequency $\omega$ s is incident together with the pump on the nonlinear crystal, the signal is amplified while the pump is depleted. At the same time, an idler wave is generated at the difference frequency $\omega_{\mathrm{i}}=\omega_{p}-\omega_{s}$ (by convention one usually assumes $\omega_{\mathrm{s}}>\omega_{\mathrm{i}}$ ). From a corpuscular point of view, this is equivalent to considering that each pump photon is split into a pair of photons respecting the conservation of energy: $\hbar \omega_{p}=\hbar \omega_{s}+\hbar \omega_{i}$.

An efficient energy transfer requires that the waves propagating along the crystal are in phase with the nonlinear polarization generating them. This is called the phase matching relation (see insert). Unlike energy conservation, phase matching does not need to be strictly
Figure 1. Major nonlinear crystals, organized according to their useful spectral window and their nonlinear figure of merit (based on data from [1]).

satisfied: it actually defines the spectral and angular acceptances of parametric interaction.

Three-wave mixing is a non-resonant, energy conservative, coherent process: phase information is preserved, and the signal and idler beams are generated at the same location while their momentums are anticorrelated in the transverse plane. From the point of view of quantum optics, the signal and idler photons are generated as a pair of entangled states. These properties have enabled

Figure 2. Drawing of a singly-resonant (left) and of a dual-cavity, doubly resonant (right) OPO.

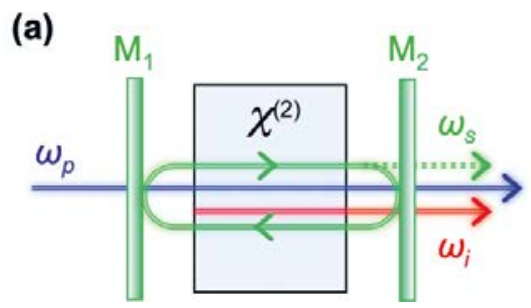

applications in phase control of optical fields, imaging with undetected photons, quantum communications and computing [2].

To a first approximation, the parametric gain is proportional to the power density (in $\mathrm{W} / \mathrm{cm}^{2}$ ) of the pump wave inside the nonlinear medium, and to the square of the nonlinear coefficient $d_{\text {eff }}$, which depends on the crystal, the direction of propagation, the wavelengths, and the type of phase matching. High gains are achieved by confining the energy in space and time, with shorter laser pulses, tightly focused laser beams, and optical cavities.

\section{OPTICAL PARAMETRIC \\ OSCILLATORS}

Similarly to a laser oscillator, it is possible to realize an optical parametric oscillator (OPO) by inserting the nonlinear crystal in an optical cavity. Parametric generation builds up on quantum noise, and the signal and idler waves are further amplified during each round trip in the optical cavity. Due to the simultaneous presence of three interacting waves, the possible configurations of optical resonators are more varied than in the case of a laser oscillator.

The closest configuration to a laser oscillator is the singly resonant OPO illustrated in figure 2(a): the mirrors of the optical cavity reflect only one of the generated waves (signal or idler), while the other is totally coupled out. This configuration has the advantage of leading to simple and robust systems. On the other hand, the oscillation threshold can be quite high (several watts in continuous-wave regime), which may require the use of a bulky pump laser.

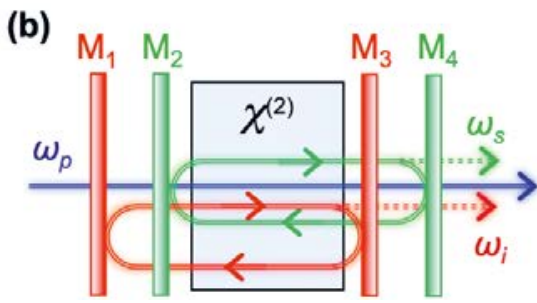


It is also possible to find a condition for which the signal and the idler waves are both resonant (see figure $2 \mathrm{~b}$ ). This is called a doubly resonant OPO. This configuration, which has no equivalent among laser oscillators, reduces the oscillation threshold by at least one order of magnitude compared to the singly resonant configuration. In the case where the two resonant waves share the same cavity, the operation may become unstable because it is necessary that the signal, $\omega_{s}$, and idler, $\omega$, frequencies each correspond to a mode of the optical resonator, while satisfying $\omega_{p}=\omega_{s}+\omega_{i}$. A solution to avoid this problem is to decouple the signal and idler cavities, e.g., by using the dual-cavity OPO scheme illustrated in figure $2 \mathrm{~b}$.

OPO cavities often require low-loss custom dielectric coatings for the mirrors and the nonlinear crystals, which generally represent a challenge for suppliers, especially in the mid-infrared. The parametric gain is directly related to the intensity of the waves that depends strongly on the duration of the pump laser pulses. This is why it is common to distinguish the applications of nonlinear optics according to the temporal regime of operation.

\section{CONTINUOUS-WAVE REGIME}

Reaching a sufficient parametric gain in the continuous-wave regime typically requires a pump laser delivering several watts in a diffraction limited beam. This is now routinely done with commercial products such as diode pumped solid state lasers and fibre lasers. Besides, the nonlinear coefficient has been increased thanks to the development of quasi-phase matched crystals such as periodically poled lithium niobate (PPLN), with the added benefit of being virtually free of adverse effects such as photorefractive focusing, grey tracking, and optical absorption.

In a continuous-wave OPO, the photon conversion efficiency is only limited by passive losses and gain saturation at the center of the Gaussian beams. It is therefore possible to approach a quantum efficiency of $100 \%$ [3]. Due to the homogeneous nature of the parametric gain (all the longitudinal modes of the cavity share the same gain), oscillation naturally converges to the longitudinal mode closest to perfect phase matching. Thereby, continuous-wave OPOs have found application in high resolution spectroscopy.

\section{PULSED REGIME}

Since parametric oscillation starts from quantum noise, several cavity roundtrips are necessary before the pulses can be detected: this is called the buildup time. During this time, amplification is very high for the oscillating pulses, but pump depletion is negligible, i.e. a significant fraction of the pump energy passes through the crystal without being converted. Hence the quantum conversion efficiency is lower than that obtained in the continuous-wave regime.

The pulsed regime has been widely used to pump OPOs because of the large availability of efficient, compact Q-switched pump lasers, emitting nanosecond or sub-nanosecond pulses with several kilowatts of peak power. The high peak power relaxes the need for a high finesse cavity, so that pulsed OPO cavities are usually shorter and more rugged than those used in other temporal regimes. Short pulse duration enables time-resolved measurement, while the Fourier-Transform-limited spectrum of nanosecond pulses is typically less than $100 \mathrm{MHz}$, which is compatible with spectroscopic analysis of gases at atmospheric pressure. Hence nanosecond OPOs have been used in telemetry, remote gas analysis, optical countermeasures, and active imaging.

In the pulsed regime, selection of a longitudinal mode by the phase matching condition cannot reach steady state. It is thus necessary to resort to line-narrowing technics to force a monochromatic spectrum. In singly resonant OPOs, the usually implemented technics are inherited from lasers; e.g., the use of an intra-cavity spectral filter or injection of narrow-linewidth continuous-wave seed radiation. In doubly resonant OPOs, a very efficient filter can be synthetized by combining the Fabry-Perot responses of signal and idler cavities of different optical lengths, a technique referred as nonlinear Vernier-effect spectral filtering (figure 3) [4].
For synchrotrons, X-FEL, HHG

- Live characterization and alignment of optics, complex optical systems \& beamlines - Phase imaging - Adaptive optics

HASO HXR 5-25 keV Single-shot Compact Up to $150 \times 150$ phase points

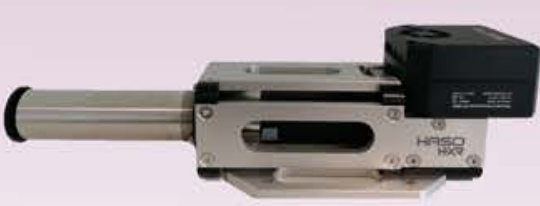

HASO EUV 30-300 eV Single-shot Vacuum compatible $72 \times 72$ phase points
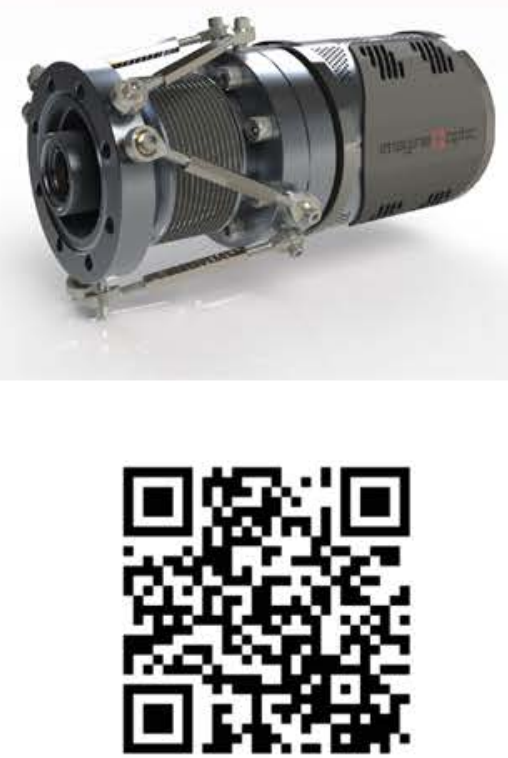

sales@imagine-optic.com

www.imagine-optic.com

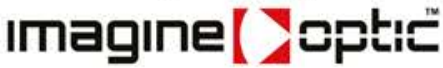




\section{ULTRA-SHORT}

\section{PULSES GENERATION}

With ultrashort pump pulses, in the picosecond and femtosecond ranges, peak intensities reach the megawatt range, enabling amplification above detectable power levels with a singlepass through the crystal. The required crystal length is typically one order of magnitude shorter than in the nanosecond range, increasing the spectral acceptance tenfold.
Thus, nonlinear crystals have been extensively used to amplify wideband ultrashort pulses, especially with a technique called optical parametric chirped-pulse amplification (OPCPA), which is the nonlinear optics analog of the chirped-pulse amplification (CPA) for which a Nobel Prize in Physics was awarded in 2018. Such ultrashort, high-intensity parametric sources are mainly developed for high-field physics [5].
Regarding parametric oscillators, since the spatial extent of ultrashort pulses is shorter than the crystal itself, amplification of light reflected by the cavity during the same pump pulse is not possible. One then has to resort to a technique called synchronous pumping, that shares similarities to gain mode locking in lasers. In this case, the round trip time in the OPO cavity is matched to the repetition rate of the

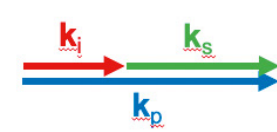

Collinear, exact PM

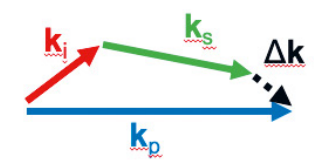

General, imperfect PM

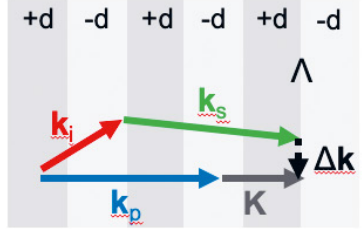

General QPM

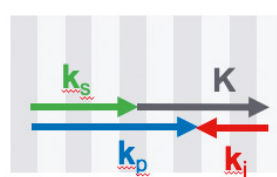

Collinear backward QPM

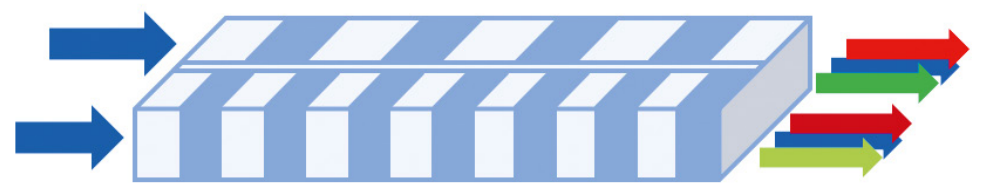

Quasi phase matching with two different periods
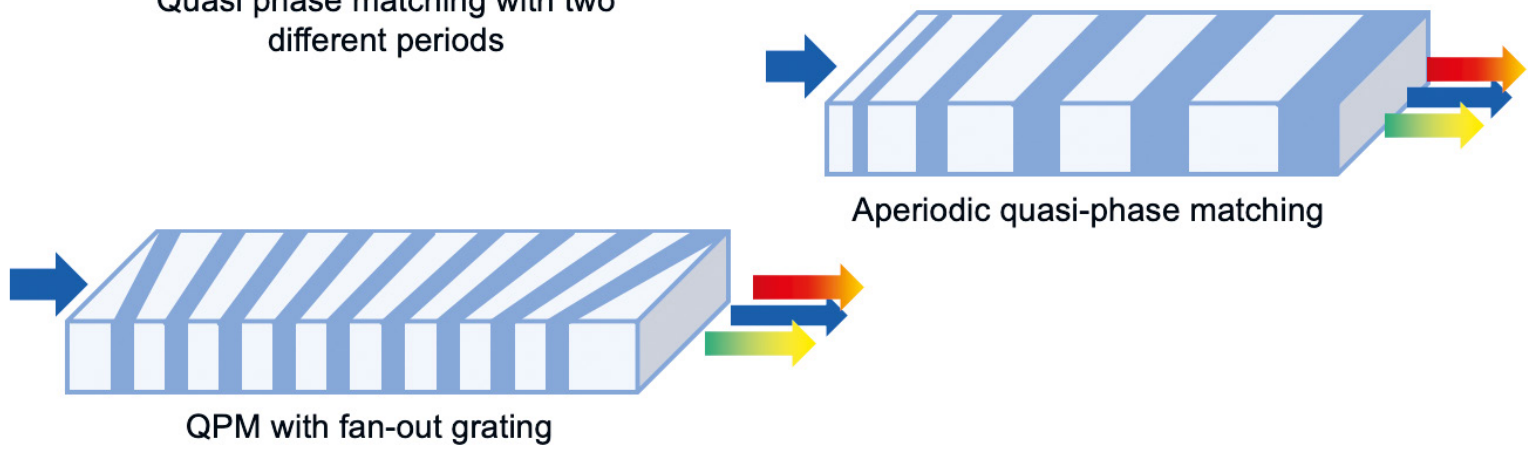

Aperiodic quasi-phase matching

The phase-matching relation usually writes: $\overrightarrow{\Delta k}=\vec{k}_{p}-\vec{k}_{s}-\vec{k}_{i} \rightarrow 0$, using the wavevectors $\vec{k}=\frac{n(\omega, \vec{u}) \omega}{c} \vec{u}$, where $\vec{u}$ is the unitary vector perpendicular to the wavefront. The maximum tolerable phase mismatch corresponds typically to $|\Delta k|_{\max }=\pi / L$, where $L$ is the length of the nonlinear medium. Because $\Delta k$ depends on the frequency through the index of refraction, it determines the spectral acceptance of the three-wave mixing process, i.e. the parametric gain bandwidth. Because of chromatic dispersion, which generally leads to $n_{p}>n_{s}, n_{i}$, exact phase matching is only possible in birefringent materials in which the dependence of the index to the direction of propagation $\vec{u}$ is used to compensate for dispersion. Another method is to use modal birefringence in waveguides. However, the most popular method is to periodically structure the nonlinear response of the material, which is usually done by electric field poling. The resulting grating of period $\Lambda$ contributes to phase matching through a wavevector $\vec{k}=\frac{2 \pi}{\Lambda} \vec{u}_{z}$. This so-called Quasi Phase Matching (QPM) method offers many more features than birefringent phase matching, because the crystallographic domains can be oriented and structured to different aims [7]: selecting the highest coefficient among the nonlinear tensor $d$, generating more than two wavelengths, shaping the gain spectrum, generating a wave in the backward direction, generating a perpendicular THz wave, etc. QPM can also be used with isotropic crystals, such as GaAs. 


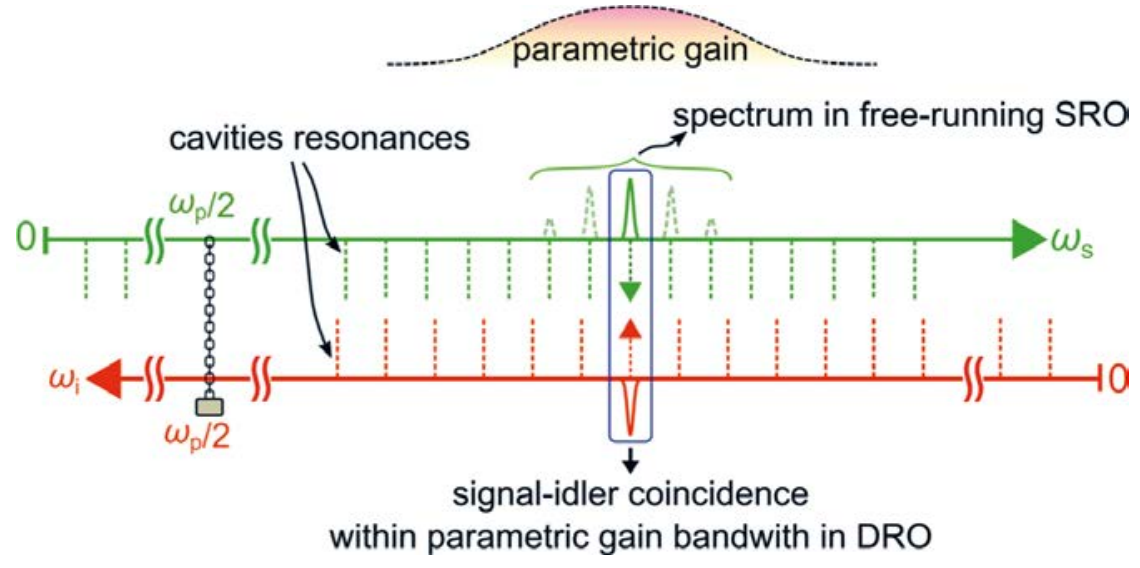

| Figure 3. Mode selection by the dual cavity scheme.

pump laser, so that the signal and/or idler pulse generated by a pump pulse is amplified by the next pump pulse after a cavity round trip. If the pump laser is a mode-locked laser, the constant phase relation between pump pulses also means that the signal/idler pulses are in phase: the OPO emits a train of mode-locked pulses at two new wavelengths. In the spectral domain, this train of mode-locked pulses is a frequency comb, at the heart of the Nobel Prize in Physics in 2005, which, thanks to the very large spectral acceptance of the parametric gain, can span more than an octave $(\omega-2 \omega)$. This property enables to measure the phase-carrier offset that defines the fundamental frequency of the comb, and stabilize it in time. This step has been the cornerstone of high resolution dual-comb spectroscopy in the mid-infrared [6].

\section{CONCLUSION}

Rephrasing Maiman's citation on lasers, we can say that the optical parametric oscillator has originally been "a solution seeking a problem". Today, it is a fundamental component for controlling the properties of coherent light in any part of the spectrum, with a sufficient optical-to-optical efficiency. Laser companies now propose products dedicated to OPO pumping. While candies can come in many flavors, OPOs can take very different forms, from table-top servo-locked cavities for demanding laboratory experiments, to semiconductor waveguides and monolithic mirrorless cavities for industrial applications. These unique properties make them highly relevant for various applications such as gas sensing or infrared countermeasures as well as for fundamental science spanning from quantum optics to strong-field physics.

\section{REFERENCES}

[1] V.G. Dmitriev, G.G.Gurzadyan, D.N. Nikogosyan, Handlbook of nonlinear optical crystals (Springer, 2013)

[2] C. Couteau, Contemp. Phys. 59, 291-304 (2018)

[3] W.R. Bosenberg,A. Drobshoff, J.I.Alexander, L.E. Myers, R.L. Byer, Opt. Lett. 21, 1336-1338 (1996) [4] A. Godard, M. Raybaut, M. Lefebvre, in Encyclopedia of Analytical Chemistry, edited by Meyers, R. A. (John Wiley \& Sons, Ltd, Chichester, UK, 2017), pp. 1-35

[5] A.G. Ciriolo et al., Appl. Sci. 7, 265 (2017)

[6] A. Schliesser, N. Picqué, T.W. Hänsch, Nat. Photonics 6, 440-449 (2012)

[7] D.S. Hum, \& M.M. Fejer, C. R. Physique 8, 180-198 (2007)

\section{A UNIQUE RANGE OF ALL SOLID-STATE TUNABLE LASERS THANKS TO LUMIBIRD \& GWU PARTNERSHIP}

\section{PULSED ND:YAGs \& OPOS}

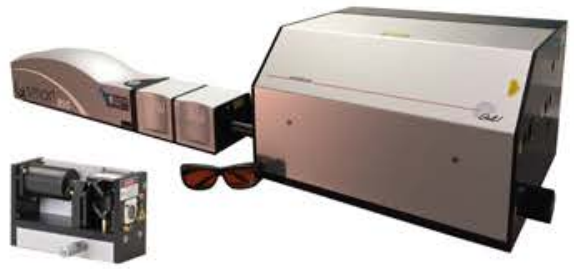

- Guaranteed compatibility \& worldwide support

- Repetition rate from $10 \mathrm{~Hz}$ to $400 \mathrm{~Hz}$

- Hands \& gap-free tunability

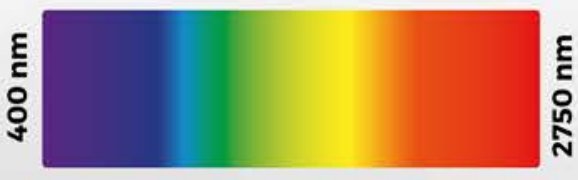

- Fast wavelength switching $<100 \mathrm{~ms}$

- Excellent beam separation contrast > 1:10 000

For photoacoustics, spectroscopy, combustion, light-matter interaction, PDT, and many more

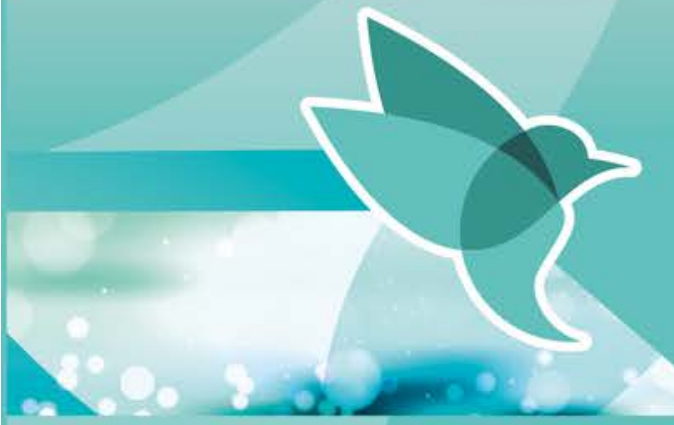

YOU HAVE A PROJECT ? CONTACT US !

www.lumibird.com

THE SPECIALIST

IN LASER TECHNOLOGIES 